\title{
Impact of Diabetes on the Left Ventricular Long Axis and Midwall Function in Patients with Hypertension
}

\author{
Jeong-Hwan Park, II-Suk Sohn*, Jin-Man Cho and Chong-Jin Kim \\ Department of Cardiology, Kyunghee East-West Neo Medical Center, Seoul, Korea
}

\begin{abstract}
Diabetes mellitus (DM) is a strong risk factor for cardiovascular morbidity and mortality. It is suggested that both hypertension and DM may synergistically affect left ventricular (LV) structure and function. To assess whether hypertensive patients with DM have more depressed LV function in terms of long axis and midwall function by use of tissue Doppler echocardiogram than do patients with hypertension only. Hypertensive patients $(n=200)$ and hypertensive patients with DM $(n=60)$, all of whom had normal LV ejection fraction, were recruited for the study. Healthy age- and sex-matched subjects $(n=20)$ served as the control group. Echocardiography was performed including LV wall thickness, dimension, Doppler, and tissue Doppler imaging. Blood pressure (BP) was measured in the supine position before and after echocardiographic examination. The hypertensive patients with or without DM had higher BP and LV wall thickness, had more decreased afterload measured by meridional end-systolic stress, and had more depressed LV diastolic, long axis, and global function than did the controls. There was no difference in LV function between the hypertensive patients with and without DM. The hypertensive patients with or without DM had depressed LV function compared with the controls, but there was no difference between the hypertensive patients with or without DM. DM does not seem to have additive adverse effects on the LV long axis and midwall function in persons with hypertension.
\end{abstract}

Key Words: Diabetes mellitus; Hypertension; Ventricular function, Left; Echocardiography

\section{Introduction}

Diabetes mellitus (DM) and hypertension both contribute to cardiovascular disease leading to atherosclerosis, ischemic heart disease, and heart failure. Hypertension is often associated with impaired glucose tolerance and insulin resistance and is not uncommonly combined with DM. ${ }^{1}$ Hypertension and DM are continuously increasing in the general population and are

Accepted for Publication: March 22, 2009

*Corresponding author: II-Suk Sohn, 134-727, Cardiovascular Center of Kyunghee East-West Neo Medical Center, Phone: +82-2-440-6108, FAX: +82-2-442-6638, E-mail: issohn@khu.ac.kr associated with adverse remodeling of the cardiovascular system. ${ }^{2}$ This high risk for progressive adverse effects on the heart from the preclinical phase has resulted in novel American College of Cardiology/ American Heart Association (ACC/AHA) guidelines for the classification of heart failure, which include stage $\mathrm{A}$ and $\mathrm{B}$ before overt heart failure symptoms develop.

DM can affect cardiac structure and function in the absence of ischemic heart disease or hypertension. ${ }^{4}$ This condition, also known as diabetic cardiomyopathy, ${ }^{5}$ is often characterized by left ventricular (LV) hypertrophy and diastolic dysfunction, which can be easily detected 
by using Doppler echocardiography. Elevated blood pressure itself typically affects the heart, and it is commonly associated with increased LV mass, LV dilatation, diastolic dysfunction, reduced chamber compliance, and progression to overt heart failure. ${ }^{6-8}$

Recent echocardiographic advances, especially in tissue Doppler imaging, can assess subtle changes in LV structure and function. This may provide useful information about diastolic function in patients with DM, hypertension, or both. ${ }^{9,10}$ However, there are few data from tissue Doppler imaging on the impact of DM on LV function in patients with hypertension. Therefore, we tried to assess whether hypertensive patients with DM have more depressed LV function in terms of long axis and midwall function by use of tissue Doppler echocardiogram than do patients with hypertension only.

\section{Materials and Methods}

Patients with hypertension who had been treated with antihypertensive drugs and who had not changed during the previous 6 months were enrolled. Excluded from the study were those (1) who had a history of previous coronary artery disease or with endovascular intervention, bypass surgery, or who had regional wall motion abnormalities; (2) who had undergone open heart surgery; (3) who had significant valvular heart disease or other structural heart diseases, including congenital heart disease, hypertrophic cardiomyopathy, dilated cardiomyopathy, or low LV ejection fraction less than 45\%; and (4) who had an electrocardiographically evident left bundle branch block, preexcitation, or atrial fibrillation.

The hypertensive patients were divided into 2 groups: the hypertension group with hypertension only and the hypertension with DM group. Age- and sex-matched healthy controls who had normal blood pressure (BP), no medical history of cardiovascular disease, a normal electrocardiogram, and no remarkable organic heart dis- ease on echocardiography were also enrolled. Demographic data were obtained before echocardiography. BP was measured in the supine position before and after echocardiographic examination. All subjects gave informed consent and all examinations were approved by the Institutional Review Board.

\section{Echocardiographic examination}

Comprehensive 2-dimensional and Doppler echocardiography using a commercial machine (iE33, Philips, Netherlands) were performed in every subject in the left lateral decubitus position. Echocardiographic measurements included end-diastolic interventricular septal thickness (IVSd), end-diastolic LV posterior wall thickness (PWT), LV end-diastolic dimension (LVDd), LV end-systolic dimension (LVDs), and LV ejection fraction by biplane Simpson's method.

LV mass, ${ }^{11}$ relative wall thickness as an index of eccentricity of LV hypertrophy, and fractional shortening (FS) were defined as follows:

$$
\begin{aligned}
\cdot \mathrm{LV} \text { mass }(\mathrm{g})= & 1.04 \times\left[(\mathrm{LVDd}+\mathrm{PWT}+\mathrm{IVSd})^{3}-\right. \\
& \left.\mathrm{LVDd}^{3}\right] \times 0.8+0.6
\end{aligned}
$$

- Relative wall thickness $=2 \times \mathrm{PWT} / \mathrm{LVDd}$

- FS $(\%)=[($ LVDd - LVDs $) / L V D d] \times 100$

LV hypertrophy was defined as LV mass index $>95$ $\mathrm{g} / \mathrm{m}^{2}$ (women) or $>115 \mathrm{~g} / \mathrm{m}^{2}$ (men).

In the presence of altered LV geometry, as in hypertension, systolic shortening measured at the level of the endocardium is often higher than predicted from the measured level of end-systolic stress (ESS). ${ }^{12}$ In contrast, when measured at the midwall level, systolic shortening is often depressed. ${ }^{13,14}$ Thus, midwall FS was calculated as described previously elsewhere. ${ }^{14}$

The force-limiting LV ejection (i.e., chamber function) is myocardial afterload, which can be measured as wall stress at end-systole according to the principles of Laplace's law. ${ }^{15}$ Longitudinal myocardial fiber shortening is limited by longitudinal (meridional) ESS. This meridional $\mathrm{ESS}^{13,16}$ was calculated as:

- Meridional ESS=0.334 $\times$ systolic blood pressure $\times$ LVDs/ $\{\mathrm{PWT} \times[1+(\mathrm{PWT} / \mathrm{LVDs})]\}$ 
Pulsed Doppler imaging from the mitral inflow measured early (E) and late (A) velocity and deceleration time (DT) of $\mathrm{E}$ velocity. Mitral annular early diastolic $\left(E^{\prime}\right)$ and systolic $\left(S^{\prime}\right)$ velocities from the medial annulus were obtained by using tissue Doppler imaging. The ratio of early mitral inflow (E) to early annular (E') velocity, as a representative parameter of LV filling pressure, was derived. The Tei index as a well-known parameter of global myocardial function was measured from pulsed Doppler imaging of transmitral inflow and LV outflow.

\section{Statistical methods}

Data are expressed as mean \pm SD. Differences between the 3 groups were tested by ANOVA for continuous variables with post-hoc analysis by Scheffe test. Pearson chi-square test was used to test for differences in categorical variables. Univariate analysis by Pearson correlation and multivariate logistic regression analyses were performed to assess the relation of clinical and echocardiographic parameters to LV diastolic function independent of established covariates. Two-tailed $p$ $<0.05$ was considered significant. All data were managed by using a commercial statistical program (SPSS for Windows, version 10.0, Chicago, Illinois, USA).

\section{Results}

\section{Clinical characteristics}

As shown in Table 1 , hypertensive patients $(n=200)$, hypertensive patients with DM $(n=60)$, and age-, and sex-matched controls $(n=20)$ were enrolled. The hypertensive patients with or without DM had higher body mass index, systolic and diastolic BP, and pulse pressure than did the healthy controls. On average, the duration of hypertension was 7.7 years in hypertension group and 6.0 years in the hypertension with DM group. The DM patients had the disease for 7 years on average. Among the antihypertensive drugs, angiotensin-converting enzyme inhibitor and/or angiotensin-receptor blocker was more commonly used in the hypertension with DM group than in the hypertension group (65\% vs $47 \%$ ). There were no differences in age, sex, current smoking, or heart rate between the study groups.

\section{Echocardiographic parameters of LV function}

The hypertensive patients with or without DM had higher IVSd, LV mass index, and relative wall thickness than did the control group, but not LV dimension, FS, or ejection fraction (Table 2). The patients with hypertension had lower meridional ESS than did the controls. Of the diastolic function parameters, DT, isovolu-

Table 1. Clinical characteristics of the subjects

\begin{tabular}{lccc}
\hline & Control $(\mathrm{n}=20)$ & Hypertension $(\mathrm{n}=200)$ & Hypertension with DM $(\mathrm{n}=60)$ \\
\hline Age (years) & $55.2 \pm 7.9$ & $56.2 \pm 8.2$ & $56.2 \pm 7.4$ \\
Female (\%) & 60 & 52 & 50 \\
Body mass index $\left(\mathrm{kg} / \mathrm{m}^{2}\right)$ & $23.2 \pm 3.2$ & $25.1 \pm 3.0^{*}$ & $25.6 \pm 3.3^{*}$ \\
Current Smoker (\%) & 20 & 21 & 20 \\
Systolic BP (mmHg) & $120.0 \pm 8.3$ & $133.1 \pm 13.2^{\dagger}$ & $136.4^{\dagger} \pm 12.6^{\dagger}$ \\
Diastolic BP (mmHg) & $74.9 \pm 5.9$ & $84.1 \pm 7.8^{\dagger}$ & $82.9 \pm 7.9^{\dagger}$ \\
Pulse pressure (mmHg) & $45.2 \pm 7.3$ & $49.0 \pm 10.9^{*}$ & $53.5 \pm 10.5^{*}$ \\
Heart rate (beats/min) & $64.9 \pm 8.1$ & $64.5 \pm 9.4$ & $66.7 \pm 10.4$ \\
Duration of hypertension (years) & & $7.7 \pm 6.6$ & $6.0 \pm 5.3^{*}$ \\
Duration of diabetes (years) & & $7.0 \pm 5.7$ \\
Antihypertensive drugs & & 47 & $65^{\ddagger}$ \\
ACEi/ARB (\%) & & 78 & 67 \\
Calcium antagonist (\%) & 51 & 37 \\
Beta-blocker (\%) & 19 & 22 \\
Diuretics (\%) & & & \\
\hline
\end{tabular}

$\mathrm{DM}$, diabetes mellitus; BP, blood pressure; ACEi/ARB, angiotensin-converting enzyme inhibitor/angiotensin receptor blocker. ${ }^{*} \mathrm{p}<0.05 \mathrm{vs}$ control, ${ }^{\dagger} p<0.01$ vs control, ${ }^{\dagger} p<0.05$ vs Hypertension. 
Table 2. Echocardiographic parameters of left ventricular function

\begin{tabular}{|c|c|c|c|}
\hline & Control $(n=20)$ & Hypertension $(n=200)$ & Hypertension with DM $(n=60)$ \\
\hline IVSd (cm) & $0.9 \pm 0.1$ & $1.1 \pm 0.2^{\dagger}$ & $1.1 \pm 0.2^{\dagger}$ \\
\hline LV end-diastolic dimension (cm) & $4.9 \pm 0.4$ & $4.9 \pm 0.5$ & $4.9 \pm 0.4$ \\
\hline LV end-systolic dimension (cm) & $3.1 \pm 0.3$ & $2.9 \pm 0.4$ & $2.9 \pm 0.3$ \\
\hline LV mass index $\left(\mathrm{g} / \mathrm{m}^{2}\right)$ & $83.8 \pm 13.7$ & $112.4 \pm 25.8^{\dagger}$ & $116.1 \pm 32.1^{\dagger}$ \\
\hline LV hypertrophy (\%) & 5.0 & $58.5^{\dagger}$ & $58.3^{\dagger}$ \\
\hline Relative wall thickness & $0.3 \pm 0.0$ & $0.4 \pm 0.1^{\dagger}$ & $0.4 \pm 0.1^{\dagger}$ \\
\hline Endocardial FS (\%) & $36.9 \pm 3.7$ & $40.2 \pm 4.9$ & $39.5 \pm 4.5$ \\
\hline Midwall FS (\%) & $21.6 \pm 3.6$ & $23.1 \pm 4.7$ & $23.0 \pm 4.4$ \\
\hline Ejection fraction (\%) & $66.4 \pm 4.6$ & $69.4 \pm 5.9$ & $69.6 \pm 5.5$ \\
\hline Meridional ESS $\left(\mathrm{kdyne} / \mathrm{cm}^{2}\right)$ & $72.6 \pm 18.5$ & $59.9 \pm 16.7^{\dagger}$ & $61.8 \pm 16.1^{*}$ \\
\hline Deceleration time (msec) & $172.0 \pm 20.9$ & $209.9 \pm 39.7^{\dagger}$ & $214.9 \pm 36.9^{\dagger}$ \\
\hline IVRT (msec) & $86.0 \pm 9.0$ & $106.3 \pm 19.4^{\dagger}$ & $108.0 \pm 17.4^{\dagger}$ \\
\hline $\mathrm{E} / \mathrm{A}$ ratio & $1.2 \pm 0.2$ & $0.9 \pm 0.2^{\dagger}$ & $0.9 \pm 0.2^{\dagger}$ \\
\hline E' velocity $(\mathrm{cm} / \mathrm{sec})$ & $8.9 \pm 1.4$ & $6.5 \pm 1.9^{\dagger}$ & $6.0 \pm 1.4^{\dagger}$ \\
\hline$S^{\prime}$ velocity $(\mathrm{cm} / \mathrm{sec})$ & $7.9 \pm 1.3$ & $7.4 \pm 1.4$ & $7.1 \pm 1.3$ \\
\hline E/E' ratio & $9.2 \pm 2.0$ & $11.8 \pm 3.7^{\dagger}$ & $12.6 \pm 3.2^{\dagger}$ \\
\hline Tei index & $0.38 \pm 0.06$ & $0.45 \pm 0.09^{\dagger}$ & $0.46 \pm 0.10^{\dagger}$ \\
\hline
\end{tabular}

DM, diabetes mellitus; IVSd, end-diastolic interventricular septal thickness; LV, left ventricular; FS, fractional shortening; ESS, end-systolic stress; IVRT, isovolumic relaxation time; E/A, ratio of mitral inflow early (E) velocity to late (A) velocity; E', early diastolic mitral annular velocity; $S$ ', systolic mitral annular velocity; $E / E$ ', ratio of mitral inflow early $(E)$ velocity to $E$ ' velocity. ${ }^{*} p<0.05$ vs control, ${ }^{\dagger} p<0.01$ vs control.

Table 3. Clinical and echocardiographic variables related to left ventricular diastolic function (E/E' ratio)

\begin{tabular}{lccrr}
\multicolumn{1}{c}{ Variables } & Univariate & & \multicolumn{1}{c}{ Multivariate } \\
\hline Age & $r$ & $P$ & 0.069 & 0.003 \\
LV mass index & 0.304 & $<0.001$ & 0.024 & $<0.001$ \\
Body mass index & 0.261 & $<0.001$ & 0.017 & 0.76 \\
Relative wall thickness & 0.097 & 0.11 & -0.721 & 0.73 \\
Systolic blood pressure & 0.178 & 0.003 & 0.008 & 0.54 \\
Diabetes mellitus & 0.136 & 0.023 & -0.43 & 0.27 \\
ACEi/ARB use & & & 0.136 & 0.71 \\
\hline
\end{tabular}

$E / E$ ', ratio of mitral inflow early $(E)$ velocity to early diastolic mitral annular ( $E$ ') velocity; $r$, Pearson correlation coefficient; $B$, logistic coefficient; $L V$, left ventricular; ACEi/ARB, angiotensin-converting enzyme inhibitor/angiotensin receptor blocker.

mic relaxation time, and $\mathrm{E} / \mathrm{E}^{\prime}$ ratio were higher in the hypertensive patients than in the control group. The hypertensive patients had lower $\mathrm{E}^{\prime}$ velocity, $\mathrm{E} / \mathrm{A}$ ratio, and Tei index than did the controls.

\section{Clinical correlates of echocardiographic parameters of LV diastolic function}

We tested univariate analysis with the clinical and echocardiographic variables that can affect the LV diastolic function (Table 3 ). E/E' ratio, as a marker of $\mathrm{LV}$ filling pressure, showed good correlations with age, systolic BP, pulse pressure, relative wall thickness, and LV mass index. On multivariate analysis among the clinical and echocardiographic parameters, age and LV mass index were independent predictors of LV diastolic dysfunction.

\section{Discussion}

In this study, we observed that the hypertensive patients with or without DM had increased LV mass and depressed diastolic, long axis, and global LV function compared with the healthy controls, but no differences were noted between the hypertension and the hypertension with DM groups. 
Increased LV mass or LV hypertrophy is a common finding in patients with hypertension and/or DM and is an independent risk factor for developing adverse cardiovascular events and progression to heart failure. ${ }^{17-20}$ High BP and obesity, which often coexist with DM, tend to be associated with preclinical cardiovascular abnormalities and may contribute to the association of diabetes with cardiovascular events. In this study, the patients with hypertension had higher body mass index, $\mathrm{BP}$, and pulse pressure than did the healthy controls. These characteristics in the hypertensives led to higher LV mass and more depressed LV function, but with preserved LV systolic function. Diastolic dysfunction with preserved LV systolic function is a common finding in patients with hypertension and DM even though they do not show any heart failure symptoms. In this study, we did not try to assess heart failure symptoms and excluded the patients with past coronary heart disease, significant arrhythmia, valvular heart disease, or systolic dysfunction. In the real world, however, there may be a much higher prevalence of overt and preclinical cardiovascular diseases in patients with hypertension and/or DM. Furthermore, those hypertensive patients with subclinical diastolic and long axis dysfunction as in this study should be managed and followed up for future adverse cardiovascular events.

In our study, there were no differences in LV function between the hypertension and the hypertension with DM groups, which suggests that DM might not have additive adverse effects on LV function in patients with hypertension. Only age and LV mass are independent predictive factors of LV diastolic function. But we should take into account multiple complicated variables in claiming a potential link to cardiac structure and function, and it is difficult to conclude such a link in this small observational study.

In conclusion, the hypertensive patients with or without DM had higher LV mass and depressed LV diastolic, long axis, and global function compared with the healthy controls, but there was no difference between the hypertension and the hypertension with DM groups.
First, this study is cross-sectional and observational and had a relatively small number of participants in a single center. Prospective studies with larger populations will be needed for determining clinical implication. Second, we did not assess more complicated clinical variables that can be associated with depressed LV function.

\section{References}

1. Gress TW, Nieto FJ, Shahar E, Wofford MR, Brancati FL. Hypertension and antihypertensive therapy as risk factors for type 2 diabetes mellitus. Atherosclerosis Risk in Communities Study. $N$ Engl J Med 2000;342:905-12.

2. Fox CS, Coady S, Sorlie PD, D’Agostino RB Sr, Pencina MJ, Vasan $\mathrm{RS}$, et al. Increasing cardiovascular disease burden due to diabetes mellitus: the Framingham Heart Study. Circulation 2007;115:1544-50.

3. Hunt SA, Abraham WT, Chin MH, Feldman AM, Francis GS, Ganiats TG, et al. ACC/AHA 2005 Guideline Update for the Diagnosis and Management of Chronic Heart Failure in the Adult: a report of the American College of Cardiology/American Heart Association Task Force on Practice Guidelines (Writing Committee to Update the 2001 Guidelines for the Evaluation and Management of Heart Failure): developed in collaboration with the American College of Chest Physicians and the International Society for Heart and Lung Transplantation: endorsed by the Heart Rhythm Society. Circulation 2005;112:e154-235.

4. Devereux RB, Roman MJ, Paranicas M, O'Grady MJ, Lee ET, Welty TK, et al. Impact of diabetes on cardiac structure and function: the strong heart study. Circulation 2000;101:2271-6.

5. Asbun J, Villarreal FJ. The pathogenesis of myocardial fibrosis in the setting of diabetic cardiomyopathy. J Am Coll Cardiol 2006;47:693-700.

6. Fouad FM, Slominski JM, Tarazi RC. Left ventricular diastolic function in hypertension: relation to left ventricular mass and systolic function. J Am Coll Cardiol 1984;3:1500-6.

7. Frohlich ED, Apstein C, Chobanian AV, Devereux RB, Dustan HP, Dzau V, et al. The heart in hypertension. N Engl J Med 1992;327:9981008.

8. Levy D, Larson MG, Vasan RS, Kannel WB, Ho KK. The progression from hypertension to congestive heart failure. JAMA 1996;275:1557-62.

9. Di Bonito P, Moio N, Cavuto L, Covino G, Murena E, Scilla C, et al. Early detection of diabetic cardiomyopathy: usefulness of tissue Doppler imaging. Diabet Med 2005;22:1720-5.

10. Wang M, Yip GW, Wang AY, Zhang Y, Ho PY, Tse MK, et al. Tissue Doppler imaging provides incremental prognostic value in patients with systemic hypertension and left ventricular hypertrophy. J Hypertens 2005;23:183-91.

11. Devereux RB, Alonso DR, Lutas EM, Gottlieb GJ, Campo E, Sachs I, et al. Echocardiographic assessment of left ventricular hypertrophy: comparison to necropsy findings. Am J Cardiol 1986;57:450-8.

12. de Simone G, Ganau A, Roman MJ, Devereux RB. Relation of left ventricular longitudinal and circumferential shortening to ejection fraction in the presence or in the absence of mild hypertension. $J$ Hyper- 
tens 1997;15:1011-7.

13. de Simone G, Devereux RB, Roman MJ, Ganau A, Saba PS, Alderman $\mathrm{MH}$, et al. Assessment of left ventricular function by the midwall fractional shortening/end-systolic stress relation in human hypertension. $J$ Am Coll Cardiol 1994;23:1444-51.

14. de Simone G, Devereux RB, Koren MJ, Mensah GA, Casale PN, Laragh $\mathrm{JH}$. Midwall left ventricular mechanics. An independent predictor of cardiovascular risk in arterial hypertension. Circulation 1996;93:25965.

15. Quinones MA, Gaasch WH, Cole JS, Alexander JK. Echocardiographic determination of left ventricular stress-velocity relations. Circulation 1975;51:689-700.

16. Reichek N, Wilson J, St John Sutton M, Plappert TA, Goldberg S, Hirshfeld JW. Noninvasive determination of left ventricular end-systolic stress: validation of the method and initial application. Circulation
1982:65:99-108

17. Casale PN, Devereux RB, Milner M, Zullo G, Harshfield GA, Pickering TG, et al. Value of echocardiographic measurement of left ventricular mass in predicting cardiovascular morbid events in hypertensive men. Ann Intern Med 1986;105:173-8.

18. Koren MJ, Devereux RB, Casale PN, Savage DD, Laragh JH. Relation of left ventricular mass and geometry to morbidity and mortality in uncomplicated essential hypertension. Ann Intern Med 1991;114:345-52.

19. Devereux RB, de Simone G, Ganau A, Roman MJ. Left ventricular hypertrophy and geometric remodeling in hypertension: stimuli, functional consequences and prognostic implications. J Hypertens Suppl 1994;12(Suppl):S117-27.

20. de Simone G, Gottdiener JS, Chinali M, Maurer MS. Left ventricular mass predicts heart failure not related to previous myocardial infarction: the Cardiovascular Health Study. Eur Heart J 2008;29:741-7. 